\title{
Diagnostic and Prognostic Value of Cell-Surface Vimentin-Positive Circulating Tumor Cells in Pediatric Sarcoma Patients
}

\section{Long Dao}

The University of Texas MD Anderson Cancer Center

\section{Dristhi Ragoonanan}

The University of Texas MD Anderson Cancer Center Izhar Batth

The University of Texas MD Anderson Cancer Center

\section{Arun Satelli}

The University of Texas MD Anderson Cancer Center

\section{Jessica Foglesong}

The University of Texas MD Anderson Cancer Center

\section{Jian Wang}

The University of Texas MD Anderson Cancer Center

\section{Wafik Zaky}

The University of Texas MD Anderson Cancer Center

\section{Jonathan Gill}

The University of Texas MD Anderson Cancer Center

\section{Diane Liu}

The University of Texas MD Anderson Cancer Center Aisha Albert

The University of Texas MD Anderson Cancer Center

\section{Nancy Gordon}

The University of Texas MD Anderson Cancer Center

\section{Winston Huh}

The University of Texas MD Anderson Cancer Center

\section{Douglas Harrison}

The University of Texas MD Anderson Cancer Center

\section{Cynthia Herzog}

The University of Texas MD Anderson Cancer Center

\section{Eugenie Kleinerman}

The University of Texas MD Anderson Cancer Center

\section{Richard Gorlick}


The University of Texas MD Anderson Cancer Center

\section{Najat Daw}

The University of Texas MD Anderson Cancer Center

Shulin Li ( $\nabla$ sli4@mdanderson.org )

The University of Texas MD Anderson Cancer Center https://orcid.org/0000-0002-7857-3242

\section{Primary research}

Keywords: Diagnostic and prognostic, cells, patients

Posted Date: August 2nd, 2021

DOl: https://doi.org/10.21203/rs.3.rs-700748/v1

License: (9) This work is licensed under a Creative Commons Attribution 4.0 International License. Read Full License 


\section{Abstract \\ Background}

Despite advances in care, the 5 year overall survival for patients with relapsed and or metastatic sarcoma remains as low as $<35 \%$. Currently, there are no biomarkers available to assess disease status in patients with sarcomas and as such, disease surveillance remains reliant on serial imaging which increases the risk of secondary malignancies and heightens patient anxiety.

\section{Methods}

Here, for the first time reported in the literature, we have enumerated the cell surface vimentin (CSV+) CTCs in the blood of 92 sarcoma pediatric and adolescent and young adult (AYA) patients as a possible marker of disease.

\section{Results}

We constructed a ROC with an AUC of 0.831 resulting in a sensitivity of $85.3 \%$ and a specificity of $75 \%$. Additionally, patients who were deemed to be CSV + CTC positive were found to have a worse overall survival compared to those who were CSV + CTC negative. We additionally found the use of available molecular testing increased the accuracy of our diagnostic and prognostic tests.

\section{Conclusions}

Our findings indicate that CSV + CTCs have both diagnostic and prognostic value and can possibly serve as a measure of disease burden.

\section{Introduction}

The current standard of care for patients with sarcoma includes molecular genetic testing. The purpose of this testing is often to obtain an accurate diagnosis of soft-tissue sarcoma. The information obtained from these tests is crucial to the clinical management of sarcoma, as it reveals the severity of the disease and can be used in prognosis. Recent research has focused on the presence of fusion genes in sarcoma, ${ }^{1}$ for instance, EWSR1 gene fusions in Ewing sarcoma, ${ }^{2}$ which are caused by genetic instability and are accompanied by a poor prognosis. Other common molecular targets include amplified or altered TP53. ${ }^{3}$ Information from molecular genetic testing could be combined with other liquid biopsy techniques to obtain a more accurate and complete picture of each individual's disease status.

Currently, a wide variety of technologies and methods are used to isolate and enumerate circulating tumor cells (CTCs). These cells can be difficult to characterize owing to their rarity; as few as 1 CTC can 
be found per $10^{9}$ blood cells. ${ }^{4}$ Many CTC capture technologies, including the FDA-approved Cellsearch are only capable of isolating CTCs that express epithelial cellular adhesion molecule (EPCAM) ${ }^{5,6}$ Sarcomas, however, do not generally express this protein; thus, their CTCs are cannot be isolated by these technologies. Other techniques used to isolate and enumerate sarcoma CTCs include size-based methods ${ }^{7,8}$; surface-enhanced Rahman spectroscopy ${ }^{9,10}$; magnetophoretic isolation $^{11}$; and an antibody panel in tandem with automated microscopy. ${ }^{12}$

Our own previous research showed that cell-surface vimentin (CSV) is a universal marker of CTCs. ${ }^{13}$ Vimentin is expressed in normal mesenchymal cells, but this expression is usually restricted to the cytoplasm rather than the cell surface, although leukocytes are capable of expressing CSV. ${ }^{14} \mathrm{We}$ developed a microfluidics system to isolate and enumerate CSV ${ }^{+}$CTCs from pediatric sarcoma patients' peripheral blood.

CTCs have emerged as a promising biomarker in other solid tumors but remain largely unexplored in the pediatric and adolescent/young adult (AYA) sarcoma population. Liquid biopsy is of particular interest for use in children because it avoids the need for repeated imaging and frequent sedation and may therefore be less traumatic for patients and their caregivers. Nonetheless, the scant few existing studies mostly focused on the detection of CTCs through the identification of specific fusion transcripts using rtPCR, a technique that is limited to patients in whom these transcripts are present. ${ }^{15,16}$ Other studies focused on EPCAM expression, which varies depending on the type of sarcoma. ${ }^{17}$ Thus, to date, there is no truly universal marker for pediatric sarcoma.

The Center for Medicare and Medicaid Services requires that in vitro diagnostic devices be utilized in laboratories that follow Good Laboratory Practices and are Clinical Laboratory Improvement Amendments (CLIA) certified. This is to ensure consistency in results. ${ }^{18}$ Reproducibility is a crucial aspect in the transition of a technology from the research laboratory to the clinic. Automation ensures that the results do not vary from technician to technician. As of this writing, no groups have applied automated technologies to either pediatric or sarcoma patients. ${ }^{19}$

In the context of pediatric and AYA sarcoma, enumeration of CTCs may provide important diagnostic and prognostic information. Automation of this process would allow diagnostic laboratories to use our technology for these purposes. Here, we present the first study isolating CTCs using automated CSV antibody capture in pediatric and AYA patients with sarcoma. The primary aim of this study was to use CSV antibody capture to isolate CTCs from patients with various sarcomas. We further demonstrate that patients with active sarcomas have more CTCs than do long-term survivors. We confirm that the isolated $\mathrm{CSV}^{+}$cells are indeed CTCs on the basis of their size, cluster formation, and expression of smooth muscle actin. We additionally demonstrate the ability of our technology to distinguish between patients with active sarcomas and long-term survivors with a high degree of sensitivity and specificity. Finally, we demonstrate, using a combination of molecular data and $\mathrm{CSV}^{+} \mathrm{CTC}$ enumeration, that the combined lack 
of $\mathrm{CSV}^{+} \mathrm{CTCs}$ and lack of gene variants, amplifications, and fusions is associated with a clear survival advantage.

\section{Materials And Methods}

\section{Patients and data}

This study was approved by the Institutional Review Board at The University of Texas at MD Anderson Cancer Center (Protocol: PA13-0014). All patients with a confirmed diagnosis of sarcoma at any disease stage who presented to our Pediatric and Adolescent and Young Adult service between January 2014 and January 2020 were eligible for enrollment. Patients with a confirmed sarcomas who had completed treatment at least 5 years before enrollment and had no evidence of disease were used as controls. Patients who had received chemotherapy within 30 days of sample collection were excluded from the analysis. Written informed consent was obtained from all the participants in this study. Peripheral whole blood samples were collected from 72 patients with active disease and 20 controls. CTCs were isolated from these samples as previously described.

\section{Manual isolation of CTCs}

Blood samples were subjected to gradient centrifugation by Ficoll-Paque (GE, Uppsala, Sweden). The buffy coat was then resuspended in $1 \mathrm{~mL}$ of RBC lysis buffer (Alfa Aesar, Haverhill, MA) for 7 minutes at room temperature. The resulting mixture was then resuspended in $2 \%$ FBS/PBS. The buffy coat was then subjected to the EasySep human CD45 Depletion Kit II (EasySep, Vancouver, Canada) for negative selection of $\mathrm{CD}_{4} 5^{+}$cells. Next, the cells were subjected to the EasySep Magnetic Isolation Kit for positive selection of $\mathrm{CSV}^{+}$cells, using $84-1$ antibody (MD Anderson, Houston, TX) to CSV.

\section{Automatic isolation of CTCs}

Blood samples were subjected to gradient centrifugation by Ficoll-Paque. The buffy coat was then resuspended in $100 \mu \mathrm{L}$ of PBS containing $2 \%$ FBS. Four microliters of leukocyte aggregation inhibitor (Abnova, Taipei, Taiwan) was added to the resuspended cells. Next, Abnova Cytoquest slides were coated with $1 \mathrm{mg} / \mathrm{mL}$ of streptavidin for $1 \mathrm{~h}$. The slides were then coated with an antibody to CSV (Abnova) for 1 $\mathrm{h}$ at room temperature, then washed 3 times with $200 \mu \mathrm{L}$ of PBS. The cell suspension was then loaded into an Abnova Cytoquest microfluidics pump and pumped through the anti-CSV antibody-coated Abnova Cytoquest slide.

\section{Staining of CTCs}

CTCs were blocked in 1\% BSA containing an FcR blocking reagent (Abnova, Taiwan) for 30 minutes at room temperature. Cells were then resuspended in $1 \%$ BSA containing FcR blocker with $25 \mu \mathrm{g} / \mathrm{mL}$ CSV conjugated to FITC (Abnova, Taiwan) and $50 \mu \mathrm{g} / \mathrm{mL}$ anti-CD45 conjugated to PE for $1 \mathrm{~h}$ (Abnova, Taiwan). Cells were then counterstained with Hoechst prior to imaging. 
Smooth muscle actin replaced CD45 staining in one instance of staining, shown in Fig. 1D. Following automatic isolation, cells were blocked in 1\% BSA containing an FcR blocking reagent for 30 minutes at room temperature. Cells were then resuspended in 1\% BSA containing FcR blocker with $25 \mu \mathrm{g} / \mathrm{mL}$ antiCSV conjugated to FITC and $20 \mu \mathrm{g} / \mathrm{mL}$ anti-Smooth Muscle Actin conjugated to PE for $1 \mathrm{~h}$ (Abcam, UK). Cells were then permeabilized with $0.25 \%$ NP40 (Amersham, Cleveland, OH) for 1 hour at room temperature. Cells were then stained with $4 \mu \mathrm{g} / \mathrm{mL}$ goat-anti rabbit IgG (Invitrogen, Waltham MA) for 1 hour. Cells were then counterstained with Hoechst prior to imaging.

\section{Imaging}

Slides were imaged on either a Keyence or a Bioview automated fluorescence microscope. Enumeration of $\mathrm{CSV}^{+}$cells was performed manually on the Keyence microscope. $\mathrm{CSV}^{+} \mathrm{CD} 45^{-}$cells larger than $10 \mu \mathrm{M}$ were deemed CTCs.

\section{Statistical analysis}

All statistical analyses were conducted using software GraphPad Prism 6 (San Diego, California) and R (R Development Core Team, Version 3.6.3). Continuous variables were summarized using means and standard deviations. Categorical variables were summarized using frequencies and percentages. Chisquared tests were used to determine differences in $\mathrm{CSV}^{+} \mathrm{CTC}$ positivity based on age, gender, and tumor types. Non-parametric two-tailed unpaired t-tests were used to compare differences in $\mathrm{CSV}^{+} \mathrm{CTC}$ per 6 $\mathrm{mL}$ of blood between groups. Wilcoxon rank-sum test was used to compare CTC counts between manual versus automated CTC capture methods, as well as between active sarcoma patients versus long-term survivors. The sensitivity and specificity of CTC counts for classifying patients with active sarcoma versus long-term survivors were assessed using receiver operating characteristic (ROC) analysis. The area under the ROC curve (AUC) was assessed and reported. The optimal cut-off points for CTC counts were obtained using the Youden Index method. Kaplan-Meier survival analyses and log-rank tests were used to compare overall survival between different groups according to presence of CTC and sarcoma-associated genetic mutations. In particular, the CTC counts were categorized as 0 (negative) vs $>0$ (positive). We also considered different groups based on the combination of CTC positivity and presence of genetic mutation, including CTC + mutation+, CTC + mutation-, CTC-mutation + and CTC-mutation-. All of the statistical tests were two-sided. A p-value less than 0.05 were considered statistically significant.

\section{Results}

\section{Patient characteristics}

This study enrolled 72 patients with sarcoma and 20 long-term survivors. The STARD reporting diagram is given in Fig. 1A. The patients' clinical characteristics and sarcoma types are summarized in Table 1. The median age of the patients was 14 years; 18 (20\%) patients were 10 years old or younger. We found no differences in CTC detection based on age, sex, or type of tumor (Table 1). 


\section{Manual and automated capture of CSV ${ }^{+}$CTCs yields similar results}

Our previous research demonstrated the utility of a CSV-targeted antibody in the isolation and identification of CTCs, in that CSV is a universal marker of CTCs. ${ }^{13,20-23}$ We initially used the manual isolation method described previously to capture and image CTCs. ${ }^{13}$ To ensure that our technology could be used in CLIA-certified labs, we developed an automated technique (Fig. 1B) to ensure reproducibility of results. In a similar manner to the manual $\mathrm{CSV}^{+} \mathrm{CTC}$ process, the automated method can capture and isolate CTCs in the blood of patients with any type of sarcoma (Fig. 1D). To ensure the consistency of these 2 sets of data, we performed statistical comparisons. As shown in Fig. 1C, the two processes did not significantly differ in their ability to isolate and enumerate CTCs.

\section{$\mathrm{CSV}^{+}$Cells are CTCs}

We previously showed that the captured $\mathrm{CSV}^{+} \mathrm{CTCs}$ are tumor cells via FISH analysis, tumor cell spike assays, and some sequencing analyses. ${ }^{13,24,25}$ To further validate these observations for this study, we previously stained the CTCs of an angiosarcoma patient with CD31, an angiosarcoma biomarker, and reinforced this argument by staining the CTCs of a patient with embryonal rhabdomyosarcoma with smooth muscle actin (Fig. 1D). ${ }^{23}$ Expression of CD31 and smooth muscle actin, respectively, confirmed that the $\mathrm{CSV}^{+}$cells observed were indeed angiosarcoma and embryonal rhabdomyosarcoma CTCs. These data, together with the oncogene amplification we reported in our previous publications, confirmed that the $\mathrm{CSV}^{+}$cells captured by our technology were indeed tumor cells. ${ }^{13,22}$

\section{$\mathrm{CSV}^{+}$CTCs are more abundant in the blood of patients with active sarcomas compared to long-term survivors}

With this information, we were able to enumerate $\mathrm{CSV}^{+} \mathrm{CTC}$ in the blood of pediatric and AYA patients with sarcoma. Sarcoma patients who were in remission for at least 5 years (long-term survivors) formed a control group. We found that patients with active sarcoma had significantly more $(p<0.0001) \mathrm{CSV}^{+}$ CTCs per $6 \mathrm{~mL}$ of blood than did long-term survivors (Fig. 2A). A range of 0 to $26 \mathrm{CSV}^{+} \mathrm{CTCs}$ per $6 \mathrm{~mL}$ of blood were found in these patients. Of the 72 patients with active sarcoma, 17 had no detectable $\mathrm{CSV}^{+}$ CTCs. Of the 20 control samples, 5 contained $\mathrm{CSV}^{+} \mathrm{CTCs}$, though the number of CTCs was low, ranging from 0 to 3 CTCs per $6 \mathrm{~mL}$ of blood.

\section{Sensitivity and specificity of CSV ${ }^{+}$CTCs for detecting sarcoma}

We next constructed a receiver operating characteristic (ROC) curve using this information (Fig. 2B). The area under the curve (AUC) of the ROC curve was 0.831 . Because the Youden $J$ value was 1 or more CTCs per $6 \mathrm{~mL}$ of blood, this level was used as the cutoff for CTC positivity. Using this cutoff value, the sensitivity and specificity of the test were $75 \%$ and $85.3 \%$, respectively. 
To further improve the test's sensitivity and specificity, we added the results of genetic biomarker testing obtained in the course of standard care. The rationale for adopting this strategy was that our recent report found that including the genetic biomarker MYCN to CSV + CTC positivity boosted the accuracy of predictions of non-relapse from $95-100 \%$ in neuroblastoma patients who were in remission and receiving maintenance therapy. ${ }^{20}$ Because it is difficult to pinpoint a single mutation as a marker of sarcoma, we used the entire panel of genetic analysis results obtained as part of the standard of care to detect its impact on the ROC of $\mathrm{CSV}^{+} \mathrm{CTCs}$. Indeed, when the ROC curve took also took the genetic mutations listed in Table 2 into account, the AUC increased to 0.875 (Fig. 2C), with maximum combined sensitivity and specificity of $75 \%$ and $91.2 \%$, respectively.

Because metastatic sarcoma with genetic mutations indicates a high disease burden, we constructed the ROC curve to compare patients with metastatic sarcoma to those for long-term survivors using the combination of genetic mutations and CSV + CTC. The AUC increased to 0.902 (Fig. 2D).

\section{$\mathrm{CSV}^{+}$CTC positivity is associated with poorer overall survival}

Because CTCs have been associated with poor overall survival, we hypothesized that the presence of $\mathrm{CSV}^{+} \mathrm{CTCs}$ is also associated with poor survival. To this end, we constructed survival curves. Figure $3 \mathrm{~A}$ shows that sarcoma patients who were $\mathrm{CSV}^{+} \mathrm{CTC}$ negative had longer overall survival durations than did patients who were $\mathrm{CSV}^{+} \mathrm{CTC}$ positive. The median overall survival time for patients who were $\mathrm{CSV}^{+} \mathrm{CTC}$ negative was undefined, as $76 \%$ of $\mathrm{CSV}^{+}$CTC-negative patients survived. Of the $\mathrm{CSV}^{+} \mathrm{CTC}^{-}$patients who did not survive, the median overall survival time was 1597 days. The median survival time for patients who were $\mathrm{CSV}^{+} \mathrm{CTC}$ positive and did not survive was 773 days. The median overall survival time for patients who were $\mathrm{CSV}^{+}$CTC positive was 1987 days.

Again, we incorporated clinical molecular testing results into the survival analysis, as this could reveal subsets of patients who require less-aggressive therapies (Fig. 3B). We excluded long-term survivors from this analysis. When accounting for genetic mutations, we again found that patients with no genetic mutations and patients with no $\mathrm{CSV}^{+} \mathrm{CTCs}$ and no sarcoma-associated genetic mutations had the longest survival times; all patients in this category survived. We additionally found that patients who were $\mathrm{CSV}^{+}$CTC negative and mutation positive had the lowest median survival time, 1096 days. CSV ${ }^{+}$CTCpositive patients without any sarcoma-associated genetic mutations had a median survival time of 1372 days, while $\mathrm{CSV}^{+}$CTC-positive patients bearing sarcoma-associated gene variants had a median survival time of 1509 days.

We additionally examined differences in CSV ${ }^{+}$CTCs per $6 \mathrm{~mL}$ of blood in patients in an attempt to determine whether we could use our test to distinguish between patients with non-metastatic vs metastatic sarcomas (Fig. 4A). However, we were unable to find any differences between the two groups in total CSV+CTCs per $6 \mathrm{~mL}$ of blood. 


\section{Discussion}

To date, this is the largest study to evaluate the significance of CTCs in pediatric and AYA patients with sarcoma. Current attempts to isolate CTCs from sarcomas such as ApoStream (Precision for Medicine) are antibody independent and focus on the physical properties of CTCs, such as their size in comparison to other blood cells and their membrane capacitance. ${ }^{26}$ However, the cells isolated by these methods may not necessarily be CTCs; regardless, sarcoma-specific staining is necessary for validation. As a result of these limitations, there remains much room for improvement in the much-needed identification of sarcoma CTCs.

Although we previously demonstrated that CSV is a universal biomarker for CTCs, the wide variety of sarcomas included in this study offers additional confirmation of CSV as a marker of sarcoma CTCs, especially in pediatric patients. ${ }^{13}$ Our finding that CSV was expressed concomitantly with CD31 and smooth muscle actin, both markers of sarcomas further confirms our belief that CSV is a CTC-specific biomarker for cancer. ${ }^{27,28}$ Furthermore, our observation of a CTC cluster confirms our understanding that the $\mathrm{CSV}^{+}$cells we are observing are indeed CTCs. Our previously published data also showed that $\mathrm{CSV}^{+}$ CTCs bear amplified oncogenes TP53, MDM2, and KRAS. ${ }^{13}$ Amplification of these genes is associated with metastasis and tumor heterogeneity in sarcoma and with a poor prognosis. ${ }^{29,30}$ In contrast, loss of CD31 expression in angiosarcomas is a sign of increased tumorigenesis and chemoresistance. ${ }^{31}$ Furthermore, CTC clusters have been found to be more metastatic than single CTCs. ${ }^{32}$ Taken together, these data indicate that $\mathrm{CSV}^{+} \mathrm{CTCs}$ may give prognostic information when examined beyond enumeration.

We observed more $\mathrm{CSV}^{+} \mathrm{CTC}$ in patients with active sarcomas than in long-term survivors. This was expected, as long-term survivors likely have no tumors from which CTCs can shed. Thus, $\mathrm{CSV}^{+} \mathrm{CTC}$ can be used as a diagnostic tool to identify patients with sarcoma. Tissue biopsy is considered the gold standard for diagnosis of sarcoma. However, it is an invasive procedure and carries risks such as contamination and needle tract seeding. ${ }^{33}$ Thus, liquid biopsy based on the detection of $\mathrm{CSV}^{+} \mathrm{CTCs}$ could be used to diagnose sarcoma without the risks associated with tissue biopsy. The combination of $\mathrm{CSV}^{+} \mathrm{CTC}$ detection with genetic testing could provide additional diagnostic and prognostic accuracy.

Our analyses used a cohort of long-term sarcoma survivors as a control group. The ROC curve was able to distinguish between patients with active sarcoma and survivors. When genetic mutations were incorporated into the model, the AUC improved. However, recurrence is a perennial concern even after remission is achieved. Multiple publications have shown that early detection of $\mathrm{CSV}^{+} \mathrm{CTC}$ can predict relapse and recurrence in various cancer types including neuroblastoma and breast, prostate, and colorectal cancers. ${ }^{20,34-36}$ Examining CSV ${ }^{+}$CTCs in the blood of sarcoma patients in remission would allow a way of predicting relapse other than the existing standard of surveillance imaging. In this way, earlier detection of disease relapse could lead to more rapid initiation of therapy and positively impact survival outcomes. 
Our automated method for isolating and enumerating $\mathrm{CSV}^{+} \mathrm{CTC}$ s showed no significant differences in the total number of CSV+ CTCs detected per $6 \mathrm{~mL}$ of blood, nor were the variances between the 2 groups significant ( $p=0.5578$ ). We chose the cutoff of 1 or more CSV ${ }^{+}$CTCs per $6 \mathrm{~mL}$ of blood to indicate positivity, as this was the value with the highest combined sensitivity and specificity. The automated procedure will also advance our goal of using $\mathrm{CSV}^{+} \mathrm{CTCs}$ as a diagnostic tool, as it is CLIA compliant.

Although there metastatic sarcomas had more CSV+CTCs per $6 \mathrm{~mL}$ of blood nonmetastatic tumors, but this difference was not statistically significant. A possible explanation for this finding may be that although the amount of $\mathrm{CSV}^{+} \mathrm{CTC}$ in the blood can be similar in patients with metastatic and nonmetastatic disease, differences in the tumor microenvironment that foster metastases may exist. Alternatively, the CTCs shed from nonmetastastic tumors may be less disposed to form tumors than are CTCs from metastatic tumors. The findings that the number of CSV+ CTCs per $6 \mathrm{~mL}$ of blood is higher in patients with active sarcomas compared to long-term suggest that these cells can potentially serve as a measure of disease burden.

We were also able to demonstrate that $\mathrm{CSV}^{+} \mathrm{CTC}$ positivity was indicative of poorer overall survival. With this information, it may be possible to tailor more aggressive upfront therapies to patients who are found to be $\mathrm{CSV}^{+}$CTC positive. When genetic testing was factored into the survival curves, we found no deaths among the patients who had no genetic risk factors and no CSV ${ }^{+} \mathrm{CTC}$. In contrast, all other patient groups had median survival times under 1600 days. Intriguingly, the $\mathrm{CSV}^{+} \mathrm{CTC}$-negative group had the lowest median survival time, 1096 days. A possible explanation for this is that this patient group had the lowest sample size, so any deaths in this group would dramatically impact overall survival times. Regardless, the fact that there were no deaths in the $\mathrm{CSV}^{+} \mathrm{CTC}$-negative group suggests that a combination of molecular testing and $\mathrm{CSV}^{+} \mathrm{CTC}$ enumeration can reveal a subset of patients that could safely undergo less-aggressive treatment. Furthermore, when we incorporated molecular testing data into our results, we found that patients who were both $\mathrm{CSV}^{+} \mathrm{CTC}^{-}$and tested negative for any variant genes had a survival advantage compared to all other groups. No members of this group died. A comprehensive panel that includes both testing for the variant genes listed in Table 2 and CSV ${ }^{+}$immune cells could allow patients with negative results on both these tests to discontinue therapy earlier, limiting both the deleterious effects of chemotherapy and patient anxiety.

In summary, this study demonstrates the utility of using CSV in pediatric and AYA patients as a biomarker to detect CTCs and the ability of $\mathrm{CSV}^{+} \mathrm{CTC}$ to distinguish long-term survivors from patients with active sarcomas. Furthermore, our findings demonstrate the prognostic value of $\mathrm{CSV}^{+} \mathrm{CTC}$, which may allow the early identification of patients who may benefit from modified therapies. While further research is needed, the enumeration of $\mathrm{CSV}^{+} \mathrm{CTC}$ in pediatric and AYA patients with sarcoma may have therapeutic and prognostic implications that may help guide patient management in the future.

\section{Declarations}


Acknowledgments

The authors would like to acknowledge Amy Ninetto for her role in editing the manuscript.

\section{Authors contributions:}

LD and DR carried out the experiments and wrote the manuscript.

IB and AS carried out the experiments.

JF, WZ, JG, AA, NG, WH, DJH, CH, EK, RG and ND developed the concept for the paper.

SL developed the concept for the paper, conceived the experiments, and edited the manuscript.

\section{Ethics Approval and Consent to Participate}

This study was approved by the Institutional Review Board at The University of Texas at MD Anderson Cancer Center (Protocol: PA13-0014). This study was performed in accordance with the Declaration of Helsinki.

\section{Consent for Publication}

Not applicable

\section{Data Availability}

Data is available from the authors upon reasonable request.

\section{Competing Interests}

The authors report no competing interests

\section{Funding Information}

This study was funded by the NIH Grant R01 EB026291

\section{References}

1. Powers MP. The ever-changing world of gene fusions in cancer: a secondary gene fusion and progression. Oncogene. 2019;38:7197-9.

2. Li X, McGee-Lawrence ME, Decker M, Westendorf JJ. The Ewing's sarcoma fusion protein, EWS-FLI, binds Runx2 and blocks osteoblast differentiation. J Cell Biochem. 2010;111:933-43.

3. Thoenen E, Curl A, Iwakuma T. TP53 in bone and soft tissue sarcomas. Pharmacol Ther. 2019;202:149-64. 
4. Nagrath S, Sequist LV, Maheswaran S, Bell DW, Irimia D, Ulkus L, Smith MR, Kwak EL, Digumarthy S, Muzikansky A, Ryan P, Balis UJ, et al. Isolation of rare circulating tumour cells in cancer patients by microchip technology. Nature 2007.

5. Allard WJ, Matera J, Miller MC, Repollet M, Connelly MC, Rao C, Tibbe AGJ, Uhr JW, Terstappen LWMM. Tumor cells circulate in the peripheral blood of all major carcinomas but not in healthy subjects or patients with nonmalignant diseases. Clin Cancer Res 2004.

6. Allard WJ, Terstappen LWMM. CCR 20th Anniversary Commentary: Paving the Way for Circulating Tumor Cells. Clin. Cancer Res.2015.

7. Hayashi M, Zhu P, McCarty G, Meyer CF, Pratilas CA, Levin A, Morris CD, Albert CM, Jackson KW, Tang CM, Loeb DM. Size-based detection of sarcoma circulating tumor cells and cell clusters. Oncotarget 2017.

8. Domingos Chinen LT, Lopes Mello CA, Abdallah EA, Ocea LMM, Buim ME, Breve NM, Gasparini JL, Fanelli MF, Paterlini-Bréchot P. Isolation, Detection, And immunomorphological characterization of circulating tumor cells (CTCs) from patients with different types of sarcoma using isolation by size of tumor cells: A window on sarcoma-cell invasion. Onco Targets Ther. 2014;7:1609-17.

9. Nima ZA, Mahmood M, Xu Y, Mustafa T, Watanabe F, Nedosekin DA, Juratli MA, Fahmi T, Galanzha El, Nolan JP, Basnakian AG, Zharov VP, et al. Circulating tumor cell identification by functionalized silver-gold nanorods with multicolor, super-enhanced SERS and photothermal resonances. Sci Rep $2014 ; 4$.

10. Kamińska A, Szymborski T, Witkowska E, Kijeńska-Gawrońska E, Świeszkowski W, Niciński K, Trzcińska-Danielewicz J, Girstun A. Detection of circulating tumor cells using membrane-based sers platform: A new diagnostic approach for 'liquid biopsy.' Nanomaterials 2019;9.

11. Mishra A, Dubash TD, Edd JF, Jewett MK, Garre SG, Karabacak NM, Rabe DC, Mutlu BR, Walsh JR, Kapur R, Stott SL, Maheswaran S, et al. Ultrahigh-throughput magnetic sorting of large blood volumes for epitope-agnostic isolation of circulating tumor cells. Proc Natl Acad Sci U S A. 2020;117:16839-47.

12. Gerdtsson E, Pore M, Thiele J-A, Gerdtsson AS, Malihi PD, Nevarez R, Kolatkar A, Velasco CR, Wix S, Singh $\mathrm{M}$, Carlsson A, Zurita AJ, et al. Multiplex protein detection on circulating tumor cells from liquid biopsies using imaging mass cytometry. Converg Sci Phys Oncol. 2018;4:015002.

13. Satelli A, Mitra A, Cutrera JJ, Devarie M, Xia X, Ingram DR, Dibra D, Somaiah N, Torres KE, Ravi V, Ludwig J, Kleinerman ES, et al. Universal marker and detection tool for human sarcoma circulating tumor cells. Cancer Res. 2014;74:1645-50.

14. Nieminen $M$, Henttinen T, Merinen M, Marttila-Ichihara F, Eriksson JE, Jalkanen S. Vimentin function in lymphocyte adhesion and transcellular migration. Nat Cell Biol 2006.

15. Avigad S, Cohen IJ, Zilberstein J, Liberzon E, Goshen Y, Ash S, Meller I, Kollender Y, Issakov J, Zaizov R, Yaniv I. The Predictive Potential of Molecular Detection in the Nonmetastatic Ewing Family of Tumors. Cancer. 2004;100:1053-8. 
16. Hoshino M, Ogose A, Kawashima H, Izumi T, Hotta T, Hatano H, Morita T, Otsuka H, Umezu H, Yanoma S, Tsukuda M, Endo N. Molecular analyses of cell origin and detection of circulating tumor cells in the peripheral blood in alveolar soft part sarcoma. Cancer Genet Cytogenet. 2009;190:75-80.

17. Tombolan L, Rossi E, Zin A, Santoro L, Bonvini P, Zamarchi R, Bisogno G. Pediatric sarcomas display a variable EpCAM expression in a histology-dependent manner. Trans/ Oncol 2020;13.

18. Rivers PA, Dobalian A, Germinario FA. A review and analysis of the Clinical Laboratory Improvement Amendment of 1988: Compliance plans and enforcement policy. Health Care Manage Rev. 2005;30:93-102.

19. Cohen EN, Jayachandran G, Gao H, Qiao W, Liu S, He J, Qiao Y, Yao L, Lin SH, Reuben JM. Enumeration and molecular characterization of circulating tumor cells enriched by microcavity array from stage III non-small cell lung cancer patients. Transl Lung Cancer Res. 2020;9:1974-85.

20. Batth IS, Dao L, Satelli A, Mitra A, Yi S, Noh H, Li H, Brownlee Z, Zhou S, Bond J, Wang J, Gill J, et al. Cell surface vimentin-positive circulating tumor cell-based relapse prediction in a long-term longitudinal study of postremission neuroblastoma patients. Int J Cancer. 2020;147:3550-9.

21. Li H, Meng QH, Noh H, Somaiah N, Torres KE, Xia X, Batth IS, Joseph CP, Liu M, Wang R, Li S. Cellsurface vimentin-positive macrophage-like circulating tumor cells as a novel biomarker of metastatic gastrointestinal stromal tumors. Oncoimmunology 2018;7.

22. Satelli A, Mitra A, Brownlee Z, Xia X, Bellister S, Overman MJ, Kopetz S, Ellis LM, Meng QH, Li S. Epithelial-mesenchymal transitioned circulating tumor cells capture for detecting tumor progression. Clin Cancer Res. 2015;21:899-906.

23. Satelli A, Brownlee Z, Mitra A, Meng QH, Li S. Circulating tumor cell enumeration with a combination of epithelial cell adhesion molecule-and cell-surface vimentin-based methods for monitoring breast cancer therapeutic response. Clin Chem. 2015;61:259-66.

24. Satelli A, Mitra A, Brownlee Z, Xia X, Bellister S, Overman MJ, Kopetz S, Ellis LM, Meng QH, Li S. Epithelial-mesenchymal transitioned circulating tumor cells capture for detecting tumor progression. Clin Cancer Res. 2015;21:899-906.

25. Li H, Meng QH, Noh H, Batth IS, Somaiah N, Torres KE, Xia X, Wang R, Li S. Detection of circulating tumor cells from cryopreserved human sarcoma peripheral blood mononuclear cells. Cancer Lett. 2017;403:216-23.

26. Balasubramanian P, Kinders RJ, Kummar S, Gupta V, Hasegawa D, Menachery A, Lawrence SM, Wang L, Ferry-Galow K, Davis D, Parchment RE, Tomaszewski JE, et al. Antibody-independent capture of circulating tumor cells of non-epithelial origin with the ApoStream® system. PLoS One 2017;12.

27. Rao P, Lahat G, Arnold C, Gavino AC, Lahat S, Hornick JL, Lev D, Lazar AJ. Angiosarcoma: A tissue microarray study with diagnostic implications. Am J Dermatopathol. 2013;35:432-7.

28. Riedlinger WFJ, Kozakewich HPW, Vargas SO. Myogenic markers in the evaluation of embryonal botryoid rhabdomyosarcoma of the female genital tract. Pediatr Dev Pathol. 2005;8:427-34.

29. Zietz C, Rössle M, Haas C, Sendelhofert A, Hirschmann A, Stürzl M, Löhrs U. MDM-2 oncoprotein overexpression, p53 gene mutation, and VEGF up- regulation in angiosarcomas. Am J Pathol. 
1998;153:1425-33.

30. Yokoyama R, Schneider-Stock R, Radig K, Wex T, Roessner A. Clinicopathologic implications of MDM2, p53 and K-ras gene alterations in osteosarcomas: MDM2 amplification and p53 mutations found in progressive tumors. Pathol Res Pract. 1998;194:615-21.

31. Venkataramani V, Kuffer S, Cheung KCP, Jiang X, Trumper L, Wulf GG, Strobel P. CD31 expression determines redox status and chemoresistance in human angiosarcomas. Clin Cancer Res. 2018;24:460-73.

32. Aceto N, Bardia A, Miyamoto DT, Donaldson MC, Wittner BS, Spencer JA, Yu M, Pely A, Engstrom A, Zhu H, Brannigan BW, Kapur R, et al. Circulating tumor cell clusters are oligoclonal precursors of breast cancer metastasis. Cell 2014.

33. Filippiadis DK, Charalampopoulos G, Mazioti A, Keramida K, Kelekis A. Bone and Soft-Tissue Biopsies: What You Need to Know. Semin Intervent Radiol. 2018;35:215-20.

34. Hall CS, Karhade M, Laubacher BA, Kuerer HM, Krishnamurthy S, DeSnyder S, Anderson AE, Valero V, Ueno NT, Li Y, Su X, Lucci A. Circulating Tumor Cells and Recurrence After Primary Systemic Therapy in Stage III Inflammatory Breast Cancer. J Natl Cancer Inst. 2015;107:djv250.

35. linuma H, Watanabe T, Mimori K, Adachi M, Hayashi N, Tamura J, Matsuda K, Fukushima R, Okinaga K, Sasako M, Mori M. Clinical Significance of Circulating Tumor Cells, Including Cancer Stem-Like Cells, in Peripheral Blood for Recurrence and Prognosis in Patients With Dukes' Stage B and C Colorectal Cancer. J Clin Oncol. 2011;29:1547-55.

36. Ma X, Xiao Z, Li X, Wang F, Zhang J, Zhou R, Wang J, Liu L. Prognostic role of circulating tumor cells and disseminated tumor cells in patients with prostate cancer: a systematic review and metaanalysis. Tumour Biol. 2014;35:5551-60.

\section{Tables}

Due to technical limitations, table 1, 2 is only available as a download in the Supplemental Files section.

\section{Figures}




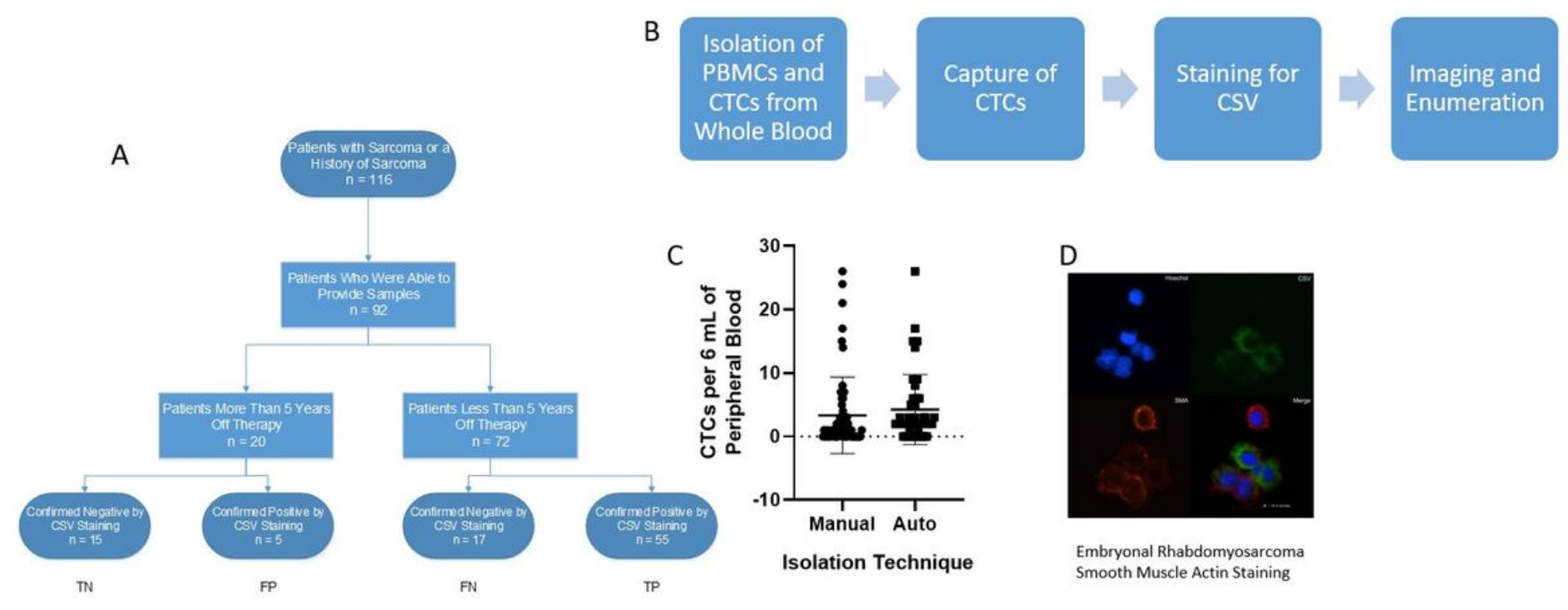

Figure 1

Isolation of CSV+CTCs from Sarcoma Patients. (a) STARD reporting diagram of consented patients. (b) Workflow diagram (c) Two-tailed t-test comparing automated vs manual technique for isolation of CTCs. $p=0.42$ (d) Representative image of CSV+ CTC. Cells were stained for nucleus (Blue), CSV (Green) and smooth muscle actin (Red) 
A

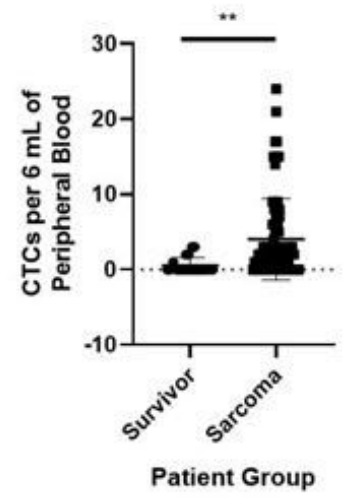

D
B

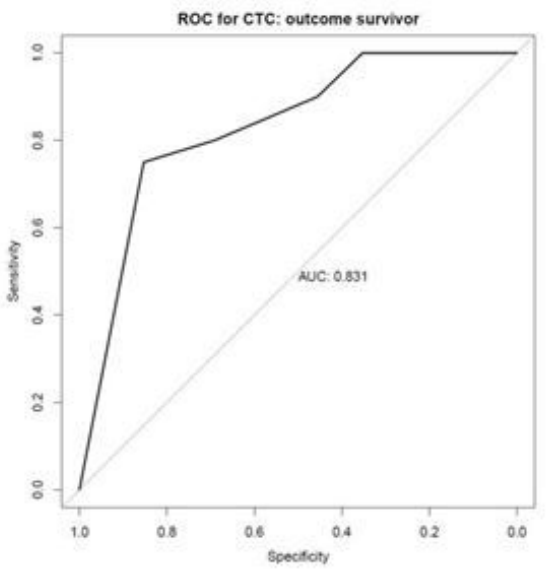

C

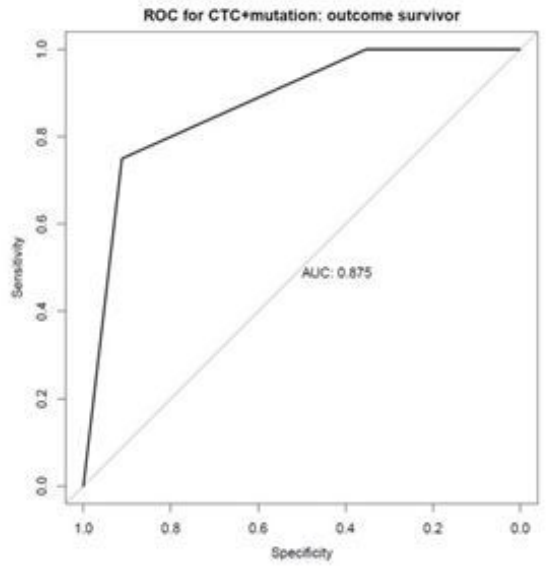

ROC for CTC+mutation: outcome Met (non survivor) vs Survivors (non Met)

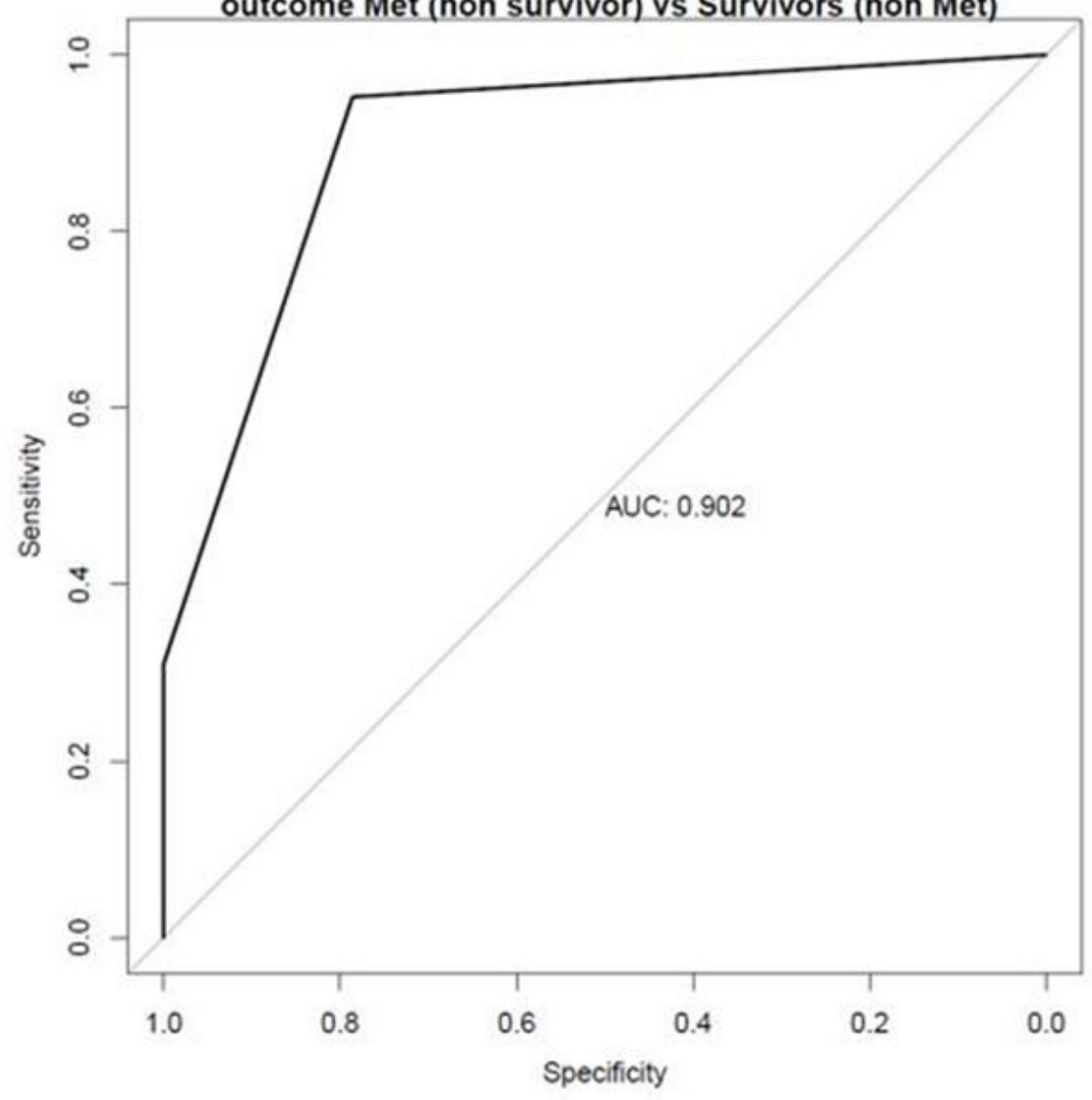

Figure 2

CSV+CTCs are elevated in the blood of patients with active sarcomas (a) Two-tailed t-test comparing CSV+CTCs per $6 \mathrm{~mL}$ of blood in long-term survivors vs patients with active sarcoma. $p<0.0001$ (b) ROC curve generated based on CSV+CTCs per $6 \mathrm{~mL}$ of blood in long-term survivors vs patients with active sarcomas (c) ROC curve generated based on CSV+CTCs per $6 \mathrm{~mL}$ of blood in long-term survivors vs patients with active sarcomas, accounting for genetic variants (d) ROC curve generated based on 
CSV+CTCs per $6 \mathrm{~mL}$ of blood in long-term survivors vs patients with active sarcomas, accounting for genetic variants and metastasis

Overall survival and CTC (all patients): Log-rank test

стс poup - cro- - cTc.

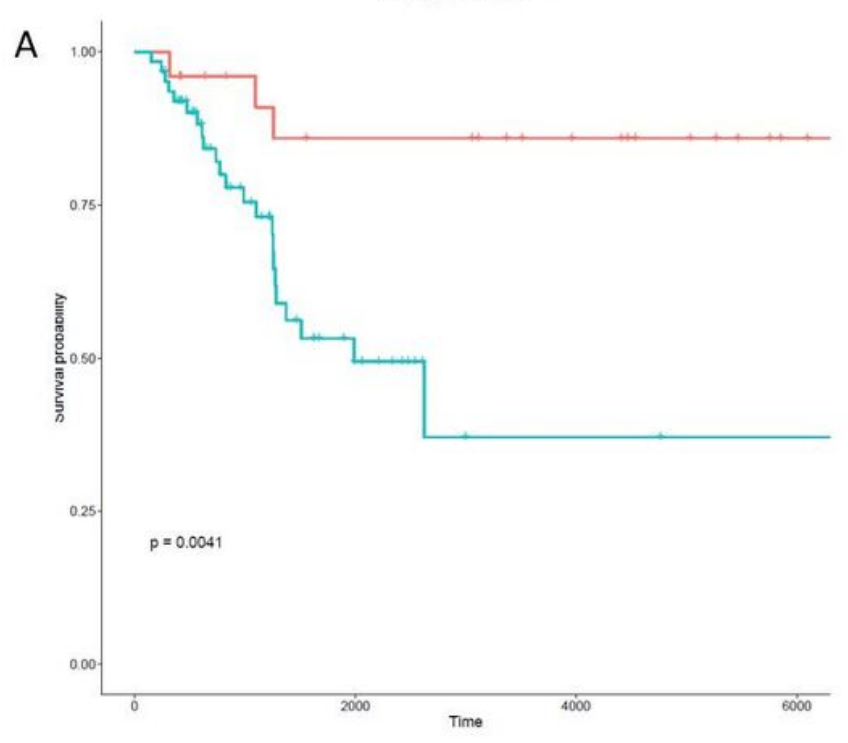

Overall survival and CTC+mutation (active sarcoma patients only): Log-rank test

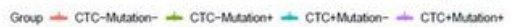

B

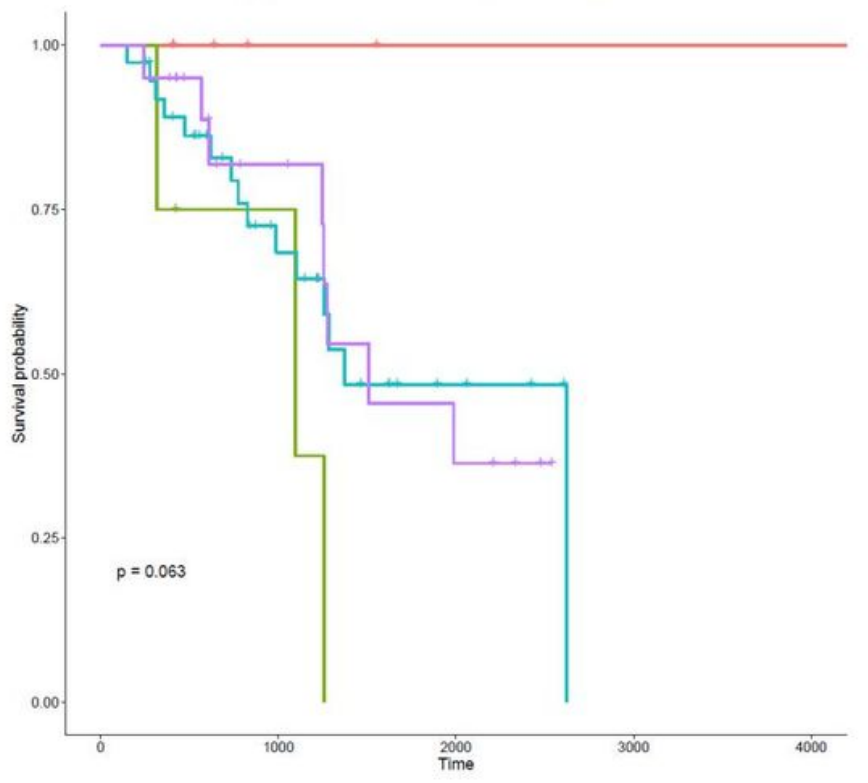

Figure 3

CSV+CTCs positivity is associated with worse prognosis (a) Kaplan-Meier survival curve of patients with $0 \mathrm{CSV}+\mathrm{CTCs}$ per $6 \mathrm{~mL}$ of blood vs patients with 1 or more CSV+CTCs per $6 \mathrm{~mL}$ of blood. $p=0.0041$ (b) Kaplan-Meier survival curve of patients with $0 \mathrm{CSV}+\mathrm{CTCs}$ with and without genetic variants versus patients with 1 or more CSV+CTCs with and without genetic variants. $p=0.063$ 


\section{Nonmetastatic vs Metastatic}

\section{A}

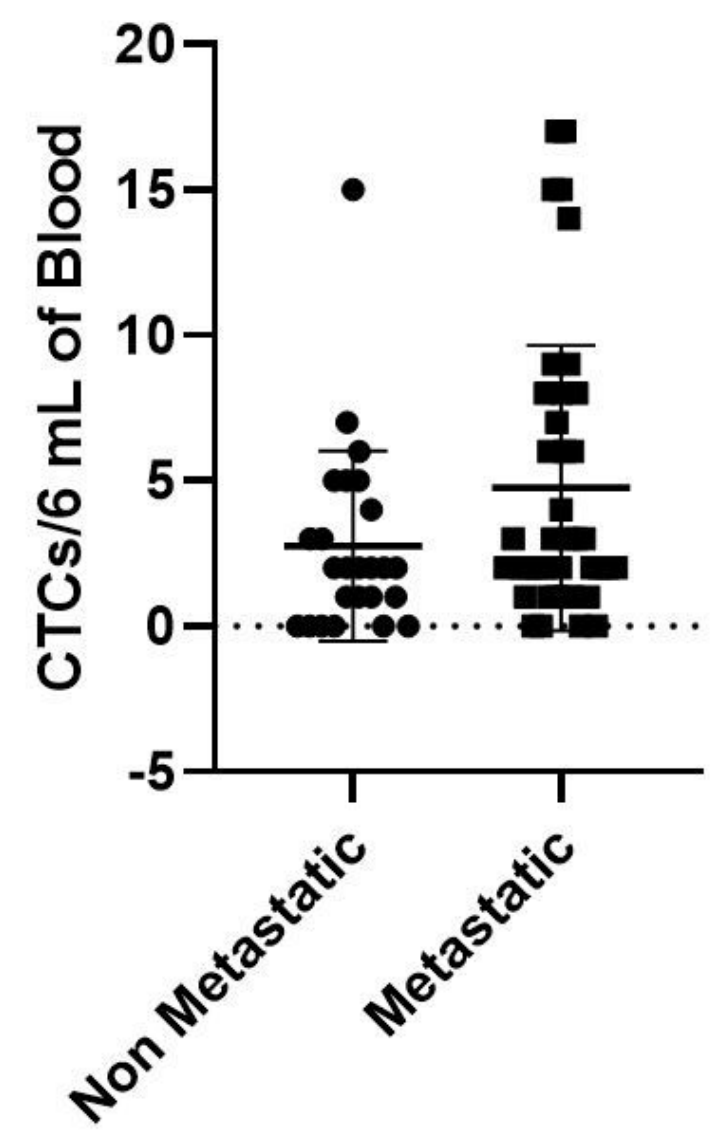

Experimental Group

Figure 4

There is no significant difference between CSV+CTCs per $6 \mathrm{~mL}$ of blood in nonmetastatic vs metastatic patients. (a) Two-tailed t-test comparing CSV+CTCs per $6 \mathrm{~mL}$ of blood in non-metastatic vs metastastic patients. $p=0.15$

\section{Supplementary Files}

This is a list of supplementary files associated with this preprint. Click to download.

- SarcomaCTCsTable1.pptx

- SarcomaCTCsTable2.pptx

- SarcomaCTCsDATAFinalMets.xIsx 